\title{
La sterilità dei dispositivi monouso rigenerati. Studio su cateteri per cardiologia interventistica dopo riutilizzo simulato
}

\author{
Iole Caola', Francesco Tessarolo', Patrizio Caciagli', Giovanni M. Guarrera ${ }^{3}$ \\ ' Laboratorio di Microbiologia e Virologia, Ospedale S. Chiara, Trento \\ ${ }^{2}$ Dip. Ingegneria dei Materiali e Tecnologie Industriali, Università di Trento \\ ${ }^{3}$ Servizio Cura e Riabilitazione, Azienda Provinciale per i Servizi Sanitari, Trento
}

Key words: Sterility, reuse, reprocessing, cardiac catheters, medical devices, Bacillus subtilis.

\section{Sterility evaluation after simulated use and reprocessing of interventional cardiology disposable catheters}

\section{SUMMARY}

The reuse of single use devices is of particular interest in interventional cardiology where the economic load of the devices represents a significant share of the whole procedure.

The sterility of the reprocessed device must be tested and warranted, together with chemical-physical and functional performances.

This study evaluates the efficiency and limitations of an experimental reprocessing protocol in order to assess the sterility of cardiac electrophysiology catheters and the maximum number of regeneration cycles sustainable by the device.

A first lot of 54 devices, collected after clinical use in the Cardiology Department of S.Chiara Hospital in Trento, was reprocessed and tested for sterility.

Devices underwent repeated cycles of simulating-use (bacteria spiked blood) and regeneration (decontamination, cleaning and hydrogen peroxide gas plasma sterilization): 36, 24, 28, 35, 22 devices were regenerated respectively 2, 3, 4, 5 and 6 times. Entire devices were cultured for 28 days in trypticase soy broth. On the whole, we tested 199 samples, six cycles of regeneration and four inoculation species. No positive sample to inoculated strain was found until the fourth cycle of reprocessing. The inoculated Bacillus subtilis strain was recovered in samples reprocessed five and six times.

Reprocessing procedures following the adopted protocol turned out efficient to guarantee device's sterility up to five reuses in experimental conditions representing the worst case scenario for bacterial load. However, complexity of reprocessing protocols, organizational issues, economic and legal requirements addressed to qualifying and certifying all steps of the reprocessing procedure discourage "in house" reprocessing.

\section{INTRODUZIONE}

La medicina moderna fa largo uso di tecnologie minimamente invasive basate su dispositivi medici monouso (SUDs), ma il crescente numero di interventi e il conseguente carico economico per il sistema sanitario, ha indotto molti paesi all'adozione di una politica di rigenerazione $(5,10$, $16,20)$.

Da un'analisi della letteratura emergono dati contrastanti circa la sicurezza e l'efficacia di un possibile riutilizzo dei SUDs $(1,2,7,9,17)$. Al giorno d'oggi, la cardiologia interventistica rappresenta uno dei campi in cui questa procedura sembra maggiormente indicata per sicurezza ed efficacia. Tuttavia vi è carenza di studi europei sulla fattibilità del riutilizzo e di convalida di procedu- re di rigenerazione.

Questa scarsità di prove tecniche e cliniche si riflette su una mancanza di approccio armonizzato della Comunità Europea nei confronti di una politica di rigenerazione.

In Italia è stata diffusa una Circolare del Ministero della Salute del 1 aprile 2005 che scoraggia la pratica di rigenerazione e riutilizzo dei dispositivi medici monouso, riconoscendo nel medesimo tempo l'assenza di un esplicito, specifico divieto nella normativa vigente in materia di dispositivi medici (4).

Per un riutilizzo dei dispositivi in condizioni di sicurezza e efficienza, la procedura di rigenerazione deve garantire il riapprontamento di tutte le caratteristiche igieniche e funzionali proprie di un disposi- 
tivo nuovo.

Si evince come sia necessario in primis fornire dettagliate linee guida che definiscano le corrette tecniche di sterilizzazione, gli adeguati controlli di qualità, e successivamente indicazioni sulle procedure organizzative e sulle responsabilità nell'utilizzo di materiali rigenerati.

La sterilità dei dispositivi medicali rappresenta la condizione igienica più stringente che deve essere garantita per un utilizzo nel paziente in condizioni sicurezza.

Gli studi disponibili in letteratura che affrontano gli aspetti microbiologici della rigenerazione di dispositivi monouso, pur affrontando le variabili critiche della procedura, presentano limitazioni quali l'incapacità di testare il dispositivo nella sua interezza, il numero di dispositivi, di cicli di rigenerazione, di specie batteriche utilizzate $(1,6,12)$ che condizionano la significatività dei risultati e la loro trasferibilità nella pratica.

In questo studio, per valutare vantaggi e limiti della procedura di rigenerazione dei cateteri per elettrofisiologia (EP), sono state sviluppate metodiche per il controllo della sterilità dei dispositivi rigenerati ad elevata sensibilità e altamente riproducibili, prestando particolare attenzione alle reali condizioni di utilizzo e rigenerazione dei dispositivi.

La procedura di rigenerazione che è stata adottata tiene conto delle caratteristiche e proprietà dei materiali, della protezione del personale coinvolto nella rigenerazione e della disponibilità di tecnologie e strutture tipiche di un'azienda ospedaliera per verificare la fattibilità di condurre la rigenerazione internamente all'ospedale.

Questo studio vede le competenze del microbiologo clinico inserite nell'interdisciplinarietà dell'approccio metodologico proprio della valutazione delle tecnologie della salute (HTA).

\section{MATERIALI E METODI}

Un lotto di 54 cateteri per elettrofisiologia cardiaca è stato raccolto nel corso di un semestre di attività nel reparto di cardiologia dell'Ospedale $\mathrm{S}$. Chiara di Trento dopo impiego clinico su paziente (tabella 1).

Tutti i dispositivi sono stati sottoposti ad una prima rigenerazione e hanno costituito un primo lotto (I lotto) di campioni.

Il protocollo di rigenerazione è stato condotto in tre fasi: decontaminazione, lavaggio (seguito da pulizia manuale con garze in tessuto non tessuto e sapone neutro) e sterilizzazione, come dettagliato in tabella 2 .

L'utilizzo simulato in vitro è stato realizzato con la contaminazione ripetuta dei dispositivi dopo il primo impiego mediante immersione in sangue inoculato con batteri.
La parte distale $(10 \mathrm{~cm})$ della sonda è stata immersa per un'ora $(6,12)$ in sangue umano intero precedentemente inoculato con una concentrazione di $10^{7} \mathrm{UFC} / \mathrm{mL}$ di uno dei seguenti ceppi batterici: Escherichia coli ATCC 11229, Bacillus subtilis ATCC 6633, Enterococcus faecium ATCC 35667 e Bacillus stearothermophilus ATCC 7953. Le specie Bacillus sono state impiegate per la loro documentata resistenza alla sterilizzazione a bassa temperatura, (14-18) utilizzando colture in cui è stata verificata la presenza sia di forme vegetative che sporali. Enterococcus faecium e Escherichia coli sono ceppi rappresentativi di batteri Grampositivi e Gram-negativi ampiamente diffusi e potenzialmente patogeni e per questo indicati in letteratura come ceppi utili alle valutazioni microbiologiche per la convalida della rigenerazione di dispositivi medici monouso (8).

Dopo la contaminazione $i$ cateteri sono stati lasciati asciugare sotto cappa senza alcun trattamento.

I lotti dal II-VI sono stati quindi ottenuti realizzando in successione le procedure di utilizzo simulato e di rigenerazione fino ad un massimo di sei cicli di rigenerazione.

Tutti i dispositivi sono stati testati in toto, inclusi la sonda catetere, il manipolo e il connettore elettrico.

\section{Test di sterilità}

Ogni dispositivo rigenerato è stato trasferito asetticamente in un cilindro sterile e immerso in circa $400 \mathrm{~mL}$ di brodo triptone soia. Il cilindro è stato sigillato con Parafilm ${ }^{\circledR} \mathrm{M}$ e trasferito in incubatore per 28 giorni a $35^{\circ} \mathrm{C}$ o a $50^{\circ} \mathrm{C}$ per i campioni contaminati con B. stearothermophilus. Se una qualunque modifica (intorbidamento, presenza di flocculazioni) era rilevata nel brodo di coltura o nel campione, un'aliquota di $5 \mathrm{~mL}$ del brodo veniva prelevata per verificare la presenza di microrganismi ed effettuare l'identificazione morfologica mediante colorazione di Gram e successiva semina in TSA $+5 \%$ sangue di montone. Le piastre venivano incubate per 24 - 48 ore e le specie isolate sono state identificate con $\mathrm{i}$ metodi tradizionali.

Le metodiche sperimentali basate sull'inoculo e incubazione per 28 giorni in un terreno di coltura non selettivo dell'intero dispositivo rappresentano una metodica sensibile e accurata per identificare eventuali dispositivi non sterili. L'elevata sensibilità della metodica impiegata tuttavia evidenzia anche contaminazioni accidentali.

Il trasferimento dei cateteri sterilizzati nel cilindro con il brodo di coltura in condizioni di asepsi (cappa a flusso laminare, guanti e camice sterile) rappresenta un passaggio critico della procedura. Sono stati registrati pertanto le possibili fonti di 
contaminazione accidentale quali errori di manipolazione e rotture della pellicola di chiusura del cilindro durante l'incubazione. Qualora i campioni interessati da errori procedurali avessero mostrato crescite di specie batteriche differenti da quelle di inoculo venivano esclusi dall'analisi statistica e dai successivi cicli di utilizzo simulatorigenerazione.

Per verificare l'assenza di sostanze inibenti nel brodo di coltura, dai cilindri senza evidenti crescite dopo 28 giorni sono state prelevate 11 aliquote da $5 \mathrm{~mL}$ ciascuna del brodo da ogni campione. 10 di esse sono state inoculate con una sospensione di $10^{3} \mathrm{UFC} / \mathrm{mL}$ dei seguenti ceppi: Staphylococcus aureus ATCC 29213, Streptococcus milleri (isolato clinico), Enterococcus faecium ATCC 35667, Streptococcus pyogenes ATCC 19615, Bacillus subtilis ATCC 6633, Escherichia coli ATCC 11229, Candida albicans (isolato clinico), Clostridium spp. (isolato clinico), Bacillus stearothermophilus ATCC 7953, Mycobacterium avium (isolato clinico). L'undicesima aliquota fungeva da controllo negativo.

I campioni che non hanno mostrato crescite di microrganismi al test di sterilità e i cui brodi di coltura risultavano non inibenti sono stati considerati sterili.

\section{RISULTATI}

Mediante la ripetizione delle fasi operative di utilizzo simulato e rigenerazione è stato possibile testare la sterilità di un totale di 199 dispositivi fino a 6 cicli di rigenerazione e 7 riutilizzi. Il numero di dispositivi sottoposti ai test di sterilità e il numero di campioni che hanno mostrato crescita della stessa specie batterica di inoculo sono riportati in tabella 3 .

L'analisi dei lotti II-VI, che comprendono i dispositivi rigenerati dopo utilizzo simulato, permette di verificare se le eventuali specie resistenti alla rigenerazione coincidano con le specie di inoculo. In tabella 3 si riportano pertanto i risultati solamente per questi lotti.

Non sono stati individuati dispositivi positivi alla specie di inoculo fino al quarto ciclo di rigenerazione, mentre nei lotti $\mathrm{V}$ e VI un dispositivo per lotto è risultato positivo alla specie $B$. subtilis, comportando una percentuale di fallimento nella rigenerazione rispettivamente di $2.9 \%(1 / 35)$ e $4.5 \%(1 / 22)$.

Tutte le aliquote prelevate dai campioni sterili hanno dimostrato una adeguata crescita batterica e hanno escluso la presenza di sostanze inibenti nei brodi impiegati che avrebbero potuto condizionare i risultati colturali.

Tabella I. Cateteri per elettrofisiologia e ablazione cardiaca sottoposti al test di sterilità

\begin{tabular}{llc}
\hline Dispositivo (modello/i) & Produttore & Numero dispositivi testati \\
\hline Marinr ${ }^{\mathrm{TM}}$ CS 7Fr 90cm & Medtronic Inc. & 8 \\
\hline Torqr $^{\mathrm{TM}}$ Josephson/Cournand type & Medtronic Inc. & 10 \\
\hline RF Conductr $^{\mathrm{TM}} 7 \mathrm{Fr}$ & Medtronic Inc. & 3 \\
\hline Stinger & BM $8 \mathrm{~mm}$ tip D/E/F Curve & 7 \\
\hline Woven $^{\mathrm{TM}} 5 \mathrm{Fr}$ Cournand type & Bard Inc. & 9 \\
\hline Compli $^{\mathrm{TM}}$ Josephson/Cournand type & Biosense-Webster Inc. & 7 \\
\hline Celsius $^{\mathrm{TM}} \mathrm{D} / \mathrm{DS}$ type & Biosense-Webster Inc. & 6 \\
\hline Avail $^{\mathrm{TM}} 6 \mathrm{Fr}$ & Biosense-Webster Inc. & 2 \\
\hline NaviStar $^{\mathrm{TM}} \mathrm{F}$ type 7Fr & Biosense-Webster Inc. & 2 \\
\hline
\end{tabular}

Tabella 2. Protocollo di decontaminazione-pulizia e sterilizzazione

\begin{tabular}{|c|c|c|c|c|}
\hline Fase & $\begin{array}{l}\text { Prodotto/Trattamento } \\
\text { (nome commerciale) }\end{array}$ & Principio attivo & Componenti principali & $\begin{array}{c}\text { Modalità di } \\
\text { utilizzo }\end{array}$ \\
\hline Decontaminazione & $\begin{array}{l}\text { Soluzione clorurata } \\
\text { (Bionil }^{\circledR} ; \text { Eurospital, Trieste) }\end{array}$ & Acido ipocloroso & $\begin{array}{l}\text { Sodio-dicloroisocianurato, } \\
\text { acido borico. }\end{array}$ & $\begin{array}{l}9.2 \mathrm{~g} / \mathrm{L} \text { in acqua } \\
(5000 \mathrm{ppm} \mathrm{di} \\
\text { cloro libero), } 10 \mathrm{~min} \text {. }\end{array}$ \\
\hline Pulizia & $\begin{array}{l}\text { Soluzione Enzimatica } \\
\text { (Septozim } \mathrm{CE}^{\circledR} ; \text { Farmec, } \\
\text { Verona) }\end{array}$ & $\begin{array}{l}\text { Proteasi alcalina, } \\
\text { tensioattivi }\end{array}$ & $\begin{array}{l}\text { Miscela enzimatica ad attività } \\
\text { proteolitica, surfattanti } \\
\text { anionici e non-ionici, } \\
\text { antischiuma siliconico }\end{array}$ & $\begin{array}{l}\text { I\% vol. in acqua } \\
\left(40-50^{\circ} \mathrm{C}\right), 10 \mathrm{~min} .\end{array}$ \\
\hline Sterilizzazione & $\begin{array}{l}\text { Sterilizzazione a gas plasma } \\
\text { (Sterrad I00S }{ }^{\circledR} \text { ASP, } \\
\text { Johnson and Johnson) }\end{array}$ & Perossido di idrogeno & $\begin{array}{l}\text { Perossido di idrogeno e } \\
\text { emissioni di radiofrequenze } \\
\text { (I3.45 MHz, 300W) }\end{array}$ & $\begin{array}{l}\text { perossido di idrogeno } \\
\text { al } 58 \% \text { con } \\
\text { ciclo breve da } 54 \mathrm{~min} \text {. }\end{array}$ \\
\hline
\end{tabular}


Tabella 3. Risultati sperimentali dei test di sterilità

\begin{tabular}{lcc}
\hline Lotto & $\begin{array}{c}\text { Dispositivi } \\
\text { testati }\end{array}$ & $\begin{array}{c}\text { Dispositivi positivi } \\
\text { al ceppo di inoculo }\end{array}$ \\
\hline I rigenerazione & 54 & N.A. \\
\hline II rigenerazione & 36 & 0 \\
\hline III rigenerazione & 24 & 0 \\
\hline IV rigenerazione & 28 & 0 \\
\hline V rigenerazione & 35 & $\mathrm{I}$ \\
\hline VI rigenerazione & 22 & $\mathrm{I}$ \\
\hline Totale & 199 & -
\end{tabular}

N.A, non applicabile. Nella colonna "dispositivi positivi vengono indicati i campioni non-sterili al test che hanno mostrato "crescita dello stesso ceppo batterico impiegato per l'utilizzo simulato. Questa classificazione non è applicabile ai campioni rigenerati dopo utilizzo su paziente e per i quali non sono noti a priori i possibili microrganismi contaminanti.

\section{DISCUSSIONE}

I risultati possono venire riassunti e discussi focalizzando due evidenze sperimentali:

i) il $100 \%$ di efficienza di sterilizzazione è stato raggiunto dopo due, tre e quattro rigenerazioni;

ii) inefficienza delle procedure di rigenerazione atte a garantire la sterilità dei dispositivi rigenerati dopo la quinta e sesta rigenerazione

Va considerato inoltre che i test di assenza di inibizione della crescita dei brodi hanno indirettamente confermato che i dispositivi non rilasciano sostanze o particolato con effetti battericidi o batteriostatici che avrebbero compromesso la validità dei test di sterilità. Il lungo periodo di incubazione è considerato sufficiente a evidenziare la presenza anche di forme batteriche a vitalità compromessa (batteri "stressati" da disinfettanti e agenti sterilizzanti), specie a lenta crescita e forme sporali. Un'ulteriore particolarità di questa metodica, se messa a confronto con altre riportate in letteratura $(1,3,6,12)$, è rappresentata dalla possibilità di testare il dispositivo nella sua integrità ed interezza. Questo risulta di fondamentale importanza considerato che, sebbene il manipolo non penetri all'interno di distretti corporei sterili, è comunque richiesta la sterilità dell'intero dispositivo per l'impiego sul campo operatorio.

L'analisi dei lotti V e VI hanno invece evidenziato crescita di $B$. subtilis e conseguente fallimento della sterilizzazione, confermando la maggiore resistenza di questa specie alla sterilizzazione a perossido di idrogeno, come già rilevato in letteratura $(14,18)$.

Comunque le alterazioni di superficie e composizione dei materiali, come gli aumenti di rugosità segnalati in seguito a procedure ripetute di rigenerazione (15) possono favorire la colonizzazione batterica (11) e diminuire l'efficienza dei protocolli di rigenerazione dopo cicli ripetuti (21).

\section{Limiti dello studio}

Le evidenze sperimentali vanno valutate tenendo in considerazione le particolari condizioni impiegate per l'utilizzo simulato, che rappresentano condizioni estreme (elevatissima carica batterica) rispetto all'impiego su paziente (pelle disinfettata, sangue sterile). Va inoltre considerato che l'incubazione prolungata in brodo di coltura, passaggio che non si trova nella ipotesi realistica, può indurre ulteriori difficoltà nella rigenerazione. Queste considerazioni possono lasciare supporre che il numero massimo di rigenerazioni sostenibili, in condizioni di sicurezza ed efficienza dal punto di vista igienico, possa essere superiore a quello sperimentale.

Infine occorre anche valutare l'efficacia del protocollo di rigenerazione adottato sia nei confronti di virus a trasmissione ematica sia per l'eliminazione dei prioni.

\section{Raccomandazioni per la rigenerazione del monouso}

I trattamenti di decontaminazione, l'immagazzinamento e il trasporto dei dispositivi durante le varie fasi del processo di rigenerazione rivestono un ruolo fondamentale al fine di conseguire il soddisfacimento delle condizioni igieniche adeguate nel dispositivo rigenerato.

Il trattamento di decontaminazione immediatamente dopo l'uso clinico deve essere ottimizzato sia per la rimozione dei residui ematici sia per la disinfezione nei confronti di batteri e virus. Oltre alle specifiche proprietà disinfettanti dei prodotti detergenti e decontaminanti è necessario considerare con attenzione le proprietà degli agenti chimici impiegati $(5,20)$. La corrosione di metalli, l'alterazione di colori e proprietà dei polimeri biomedicali possono essere correlate ad una incorretta interazione tra materiali e agenti disinfettanti.

Il lavaggio con azione meccanica, assistito dall'impiego di un efficace agente detergente e disinfettante, è particolarmente rilevante per ridurre il carico microbiologico iniziale e quindi assicurare l'efficacia sterilizzante della tecnologia Sterrad $^{\circledR}$ $(13,19)$. Il corretto precondizionamento dei dispositivi prima della sterilizzazione assicura una più efficiente penetrazione del vapore di perossido di idrogeno in tutte le aree critiche del dispositivo garantendo così il raggiungimento dello stato di sterilità.

Allo stesso tempo, la contaminazione batterica del presidio prima della sterilizzazione deve essere limitata al minimo mediante l'impiego di acqua purificata o sterile per i risciacqui e tessuti sterili o aria microfiltrata per la pulizia e l'asciugatura. Infine nell'immagazzinamento dei dispositivi parzialmente processati vanno considerati attentamente parametri determinanti quali tempo e temperatura, che devono venire ridotti il più possibile. 
Un approccio alla rigenerazione secondo tali raccomandazioni, se da un lato garantisce le migliori performance del processo, dall'altro richiede infrastrutture dedicate esclusivamente a tale processo, personale e competenze specifiche. Risulta in ogni caso irrinunciabile la necessità di garantire una procedura di rigenerazione certificata con standard qualitativi almeno equivalenti a quelli sostenuti dai produttori di dispositivi nuovi. Questi requisiti possono essere difficilmente garantiti all'interno di strutture ospedaliere mentre possono risultare maggiormente conseguibili in industrie specializzate nella rigenerazione del monouso.

Va infine considerato che i risparmi derivanti dall'introduzione di una politica di rigenerazione del monouso in cardiologia interventistica aumentano sensibilmente con l'aumentare dei dispositivi impiegati. Di contro, l'ammortamento dei costi fissi per procedure di rigenerazione condotte internamente alla struttura ospedaliera può risultare sfavorevole per un numero di interventi clinici limitato.

Aver condotto lo studio con un approccio che ha considerato:

a) la numerosità significativa del campione,

b) l'interezza e l'integrità funzionale dei dispositivi,

c) la complessa tipologia di test microbiologici effettuati,

ha permesso di aggiungere conoscenze sull'articolato processo di rigenerazione e ci auguriamo possa costituire un contributo scientifico utile a meglio comprendere i molteplici aspetti della rigenerabilità dei dispositivi monouso.

\section{RINGRAZIAMENTI}

Gli autori ringraziano per la fattiva collaborazione nella realizzazione dello studio il dott. Marcello Disertori, direttore del reparto di Cardiologia dell' ospedale S. Chiara di Trento, il Servizio infermieristico e la Centrale di Sterilizzazione per il trattamento dei dispositivi.

Lo studio è stato finanziato dal Progetto SICCSIX (Sicurezza in Cardiologia Interventistica e Cardiochirurgia), Fondo Unico 2001, Provincia Autonoma di Trento.

\section{BIBLIOGRAFIA}

1. Aton EA, Murray P, Fraser V, Coaway L, Cain ME. Safety of reusing cardiac electrophysiology catheters. Am J Cardiol 1994; 74: 1173-5.

2. Avitall B, Khan M, Krum D, Jazayeri M, Hare J. Repeated use of ablation catheters: a prospective study. J Am Coll Cardiol 1993; 22: 1367-72.

3. Bathina MN, Mickelsen S, Brooks C, Jaramillo J, Hepton T, Kusumoto F M. Safety and efficacy of hydrogen peroxide plasma sterilization for repeated use of electrophysiology catheters. J Am Coll Cardiol 1998; 32: 1384-8.

4. Circolare Ministero della Salute - Dipartimento dell'Innovazione D.G. dei Farmaci e Dispositivi Medici - DGFDM.III/P/9773/I.1.c.r.1 del 1 aprile 2005.

5. Conseil d'évaluation des technologies du Québec. The reuse of single-use cardiac catheters: safety economical ethical and legal issues. Can J Cardiol 1994; 10: 413-21.

6. Druce JD, Russell JS, Birch CJ, Yates LA, Harper RW, Smolich JJ. A decontamination and sterilization protocol employed during reuse of cardiac electrophysiology catheters inactivates human immunodeficiency virus. Infect Control Hosp Epidemiol 2003; 24: 184-90.

7. Dunnigan A, Roberts C, McNamara M, Benson DW, Benditt DG. Success of re-use of cardiac electrode catheters. Am J Cardiol 1987; 60: 807-10.

8. Fecht G. Requirements for reprocessing single-use medical devices. Zentralsterilization 1998; 6: 12-28

9. Ferrell M, Wolf CE, Ellenbogen KA, Wood MA, Clemo HF, Gilligan DM. Ethylene oxide on electrophysiology catheters following resterilization: implications for catheter reuse. Am J Cardiol 1997; 80: 1558-61.

10. Food and Drug Administration. Reuse of medical disposable devices. Compliance policy guide no 712416. Washington: Food and Drug Administration, 1987.

11. Francois P, Vaudaux P, Foster TJ, Lew DP. Host-bacteria interactions in foreign body infections. Infect Control Hosp Epidemiol 1996; 17: 514-20. Review.

12. Grabsch EA, Grayson ML, Johnson PDR, Yates LA, Harper RW, Smolich JJ. Bactericidal efficacy of sterilizing protocol for reused cardiac electrophysiology catheters. Am J Cardiol 2002; 89: 770-2.

13. Julich WD, Woedtke T. Reprocessing of thermosensitive materials-efficacy against bacterial spores and viruses. J Hosp Infect 2001; 48: 69-79.

14. Krebs MC, Becasse P, Verjat D, Darbord JD. Gas-plasma sterilization: relative efficacy of the hydrogen peroxide phase compared with that of the plasma phase. Int J Pharm 1998; 160: 75-81.

15. Lerouge S, Guignot C, Tabrizian M, Ferrier D, Yagoubi N, Yahia L. Plasma-based sterilization: effect on surface and bulk properties and hydrolytic stability of reprocessed polyurethane electrophysiology catheters. J Biomed Mater Res 2000; 15: 774-82.

16. Mickelsen S, Mickelsen C, MacIndoe C, et al. Trends and patterns in electrophysiologic and ablation catheter reuse in the United States. Am J Cardiol 2001; 87: 351-3.

17. O'Donoghue S, Platia EV. Reuse of pacing catheters: a survey of safety and efficacy. PACE 1988; 11: 1279-80.

18. Penna TCV, Ferraz AAM, Cassola MA. The presterilization load on used medical devices and the effectiveness of hydrogen peroxide gas plasma against 
Bacillus subtilis spores. Infec Control Hosp Epidemiol 1999; 20: 465-72.

19. Penna TCV, Ferraz AAM. Cleaning of blood-contaminated reprocessed angiografic catheters and spinal needles. Infec Control Hosp Epidemiol 2000; 21: 499504.

20. Robert Kock Institute recommendations. Hygienic requirements for processing of medical devices. Bundesgesundheitsblatt - GesundheitsforschungGesundheitsshutz 2001; 44: 1115-26.

21. Tessarolo F, Ferrari P, Bortoluzzi S, et al. Evaluation and quantification of reprocessing modification in single use devices in interventional cardiology. Appl Surface Sci 2004; 238: 341-6

Iole Caola

Laboratorio di Microbiologia e Virologia Ospedale S. Chiara

Largo Medaglie d'Oro 38100, Trento

Tel. 0461 903270; Fax 0461903615 e-mail: iole.caola@apss.tn.it 